\title{
Inverse nodal problem for $p$-Laplacian energy-dependent Sturm-Liouville equation
}

\section{Hikmet Koyunbakan*}

\section{"Correspondence:}

hkoyunbakan@gmail.com

Department of Mathematics, Firat

University, Elazig, 23119, Turkey

Department of Mathematics and

Statistics, Missouri University of

Science and Technology, Rolla, MO, USA

\begin{abstract}
In this study, the inverse nodal problem is solved for $p$-Laplacian Schrödinger equation with energy-dependent potential function with the Dirichlet conditions. Asymptotic estimates of eigenvalues, nodal points and nodal lengths are given by using Prüfer substitution. Especially, an explicit formula for a potential function is given by using nodal lengths. Results are more general than the classical $p$-Laplacian Sturm-Liouville problem. For the proofs, methods previously developed by Law et al. and Wang et al., in 2009 and 2011, respectively, are used. In there, they solved an inverse nodal problem for the classical $p$-Laplacian Sturm-Liouville equation with eigenparameter boundary conditions.
\end{abstract}

MSC: 34A55; 34L20

Keywords: Prüfer substitution; inverse nodal problem; $p$-Laplacian equation

\section{Introduction}

Consider the following $p$-Laplacian eigenvalue problem for

$$
-\left[\left(u^{\prime}\right)^{(p-1)}\right]^{\prime}=(p-1)(\lambda-q(x)) u^{(p-1)}, \quad 0<x<1,
$$

with the boundary conditions

$$
u(0)=u(1)=0,
$$

where $q \in L^{2}(0,1)$ is a real-valued function, $p>1$ is a constant, $u^{(p-1)}:=|u|^{p-1} \operatorname{Sgn} u$ and $\lambda$ is the spectral parameter [1]. Equation (1.1) is also known as a one-dimensional $p$-Laplacian eigenvalue equation. Note that when $p=2$, equation (1.1) becomes a Sturm-Liouville equation as

$$
-u^{\prime \prime}+q u=\lambda u
$$

and the inverse problem described in (1.1), (1.2) in the [1-8].

The determination of the form of a differential operator from spectral data associated with it has enjoyed close attention from a number of authors in recent years. One such operator is the Sturm-Liouville operator. In the typical formulation of the inverse SturmLiouville problem, one seeks to recover both $q$ and constants by giving the eigenvalues with another piece of spectral data. These data can take several forms, leading to many versions

\section{Springer}

O2013 Koyunbakan; licensee Springer. This is an Open Access article distributed under the terms of the Creative Commons Attribution License (http://creativecommons.org/licenses/by/2.0), which permits unrestricted use, distribution, and reproduction in any medium, provided the original work is properly cited. 
of the problem. Especially, the recent interest is a study by Hald and McLaughlin $[9,10]$ wherein the given spectral information consists of a set of nodal points of eigenfunctions for the Sturm-Liouville problems. These results were extended to the case of problems with eigenparameter-dependent boundary conditions by Browne and Sleeman [11]. On the other hand, Law et al. [12], Law and Yang [13] solved the inverse nodal problem of determining the smoothness of the potential function $q$ of the Sturm-Liouville problem by using nodal data. In the past few years, the inverse nodal problem of Sturm-Liouville problem has been investigated by several authors [11, 14-16].

When $q=0$, consider the problem

$$
-\left(u^{(p-1)}\right)^{\prime}=(p-1) \lambda u^{(p-1)}, \quad u(0)=u(1)=0 .
$$

The eigenvalues of this problem were given as [1]

$$
\lambda_{n}=\left(n \pi_{p}\right)^{p}, \quad n=1,2,3, \ldots,
$$

where

$$
\pi_{p}=2 \int_{0}^{1} \frac{d t}{\left(1-t^{p}\right)^{1 / p}}=\frac{2 \pi}{p \sin \left(\frac{\pi}{p}\right)}
$$

and an associated eigenfunction is denoted by $S_{p} . S_{p}$ and $S_{p}^{\prime}$ are periodic functions satisfying the identity

$$
\left[S_{p}(x)\right]^{p}+\left[S_{p}^{\prime}(x)\right]^{p}=1
$$

for arbitrary $x \in \mathbb{R}$. These functions are known as generalized sine and cosine functions and for $p=2$ become sine and cosine [17].

Now, we present some further properties of $S_{p}$ for deriving more detailed asymptotic formulas. These formulas are crucial in the solution of our problem.

\section{Lemma $1.1[1]$}

(a) For $S_{p}^{\prime} \neq 0$,

$$
\left(S_{p}^{\prime}\right)^{\prime}=-\left|\frac{S_{p}}{S_{p}^{\prime}}\right|^{p-2} S_{p}
$$

(b)

$$
\left(S_{p} S_{p}^{(p-1)}\right)^{\prime}=\left|S_{p}^{\prime}\right|^{p}-(p-1)\left|S_{p}\right|^{p}=1-p\left|S_{p}\right|^{p}=(1-p)+p\left|S_{p}^{\prime}\right|^{p} .
$$

According to the Sturm-Liouville theory, the zero set $X_{n}=\left\{x_{j}^{(n)}\right\}_{j=1}^{n}$ of the eigenfunction $u_{n}$ corresponding to $\lambda_{n}$ is called the nodal set and $l_{j}^{n}=x_{j+1}^{n}-x_{j}^{n}$ is defined as the nodal length of $u_{n}$. Using the nodal data, some uniqueness results, reconstruction and stability of potential functions have been obtained by many authors $[9,11,14-16,18]$.

Consider the $p$-Laplacian eigenvalue problem

$$
-\left[\left(u^{\prime}\right)^{(p-1)}\right]^{\prime}=(p-1)\left[\lambda^{2}-q(x)-2 \lambda r(x)\right] u^{(p-1)}, \quad 0<x<1,
$$


with the Dirichlet conditions

$$
u(0)=u(1)=0,
$$

or with the Neumann boundary conditions

$$
u^{\prime}(0)=u^{\prime}(1)=0,
$$

where $q \in L^{2}(0,1)$ and $r \in W_{2}^{1}(0,1)$ are real-valued functions, $p>1$ is a constant, $u^{(p-1)}:=$ $|u|^{p-1} \operatorname{Sgn} u$ and $\lambda$ is the spectral parameter.

In this paper, the function $r$ is known a priori and we try to construct the unknown function $q$ by the dense nodal points in the interval considered.

For $p=2$, equation (1.5) becomes

$$
-u^{\prime \prime}+[q+2 \lambda r] u=\lambda^{2} u .
$$

This equation is known as the diffusion equation or quadratic of differential pencil. Eigenvalue equation (1.6) is important for both classical and quantum mechanics. For example, such problems arise in solving the Klein-Gordon equations, which describe the motion of massless particles such as photons. Sturm-Liouville energy-dependent equations are also used for modelling vibrations of mechanical systems in viscous media (see [19]). We note that in this type of problem the spectral parameter $\lambda$ is related to the energy of the system, and this motivates the terminology 'energy-dependent' used for the spectral problem of the form (1.6). Inverse problems of quadratic pencil have been solved by many authors in the references $[15,16,18,20-27]$.

As in the $p$-Laplacian Sturm-Liouville problem, for $q=r=0$, eigenvalues of the problem given by (1.3), (1.4) are of the form

$$
\lambda_{n}=\left(n \pi_{p}\right)^{p}
$$

and associated eigenfunctions are denoted by $S_{p}$.

This paper is organized as follows. In Section 2, we give asymptotic formulas for eigenvalues, nodal points and nodal lengths. In Section 3, we give a reconstruction formula for differential pencil by using nodal parameters.

\section{Asymptotic estimates of nodal parameters}

In this section, we study the properties of eigenvalues of $p$-Laplacian operator (1.3) with Dirichlet conditions (1.4). For this, we introduce Prüfer substitution. One may easily obtain similar results for Neumann problems.

We define a modified Prüfer substitution

$$
\begin{aligned}
& u(x)=c(x) S_{p}\left(\lambda^{2 / p} \theta(x)\right), \\
& u^{\prime}(x)=\lambda^{2 / p} c(x) S_{p}^{\prime}\left(\lambda^{2 / p} \theta(x)\right)
\end{aligned}
$$

or

$$
\frac{u^{\prime}(x)}{u(x)}=\lambda^{2 / p} \frac{S_{p}^{\prime}\left(\lambda^{2 / p} \theta(x)\right)}{S_{p}\left(\lambda^{2 / p} \theta(x)\right)}
$$


Differentiating both sides of equation (2.2) with respect to $x$ and applying Lemma 1.1, one obtains that

$$
\theta^{\prime}=1-\frac{q}{\lambda^{2}} S_{p}^{p}-\frac{2}{\lambda} r S_{p}^{p}
$$

Theorem 2.1 The eigenvalues $\lambda_{n}$ of the Dirichlet problem given in (1.3), (1.4) have the form

$$
\lambda_{n}^{2 / p}=n \pi_{p}+\frac{1}{p\left(n \pi_{p}\right)^{p-1}} \int_{0}^{1} q(t) d t+\frac{2}{p\left(n \pi_{p}\right)^{\frac{p-2}{p}}} \int_{0}^{1} r(t) d t+O\left(\frac{1}{n^{\frac{p+2}{p}}}\right) .
$$

Proof For problem (1.3), (1.4), let $\lambda=\lambda_{n}, \theta(0)=0$ and $\theta(1)=\frac{n \pi_{p}}{\lambda_{n}^{2 p}}$. Firstly, we integrate both sides of $(2.3)$ over the interval $[0,1]$ :

$$
\frac{n \pi_{p}}{\lambda_{n}^{2 / p}}=1-\frac{1}{\lambda_{n}^{2}} \int_{0}^{1} q(t) S_{p}^{p}(t) d t-\frac{2}{\lambda_{n}} \int_{0}^{1} r(t) S_{p}^{p}(t) d t .
$$

Using the identity

$$
\frac{d}{d t}\left[S_{p}\left(\lambda_{n}^{2 / p} \theta(t)\right) S_{p}^{\prime}\left(\lambda_{n}^{2 / p} \theta(t)\right)^{p-1}\right]=\left(1-p\left|S_{p}\left(\lambda_{n}^{2 / p} \theta(t)\right)\right|^{p}\right) \lambda_{n}^{2 / p} \theta^{\prime}(t)
$$

and Lemma 1.1(b), we get

$$
\begin{aligned}
\frac{n \pi_{p}}{\lambda_{n}^{2 / p}}= & -\frac{1}{\lambda_{n}^{2} p} \int_{0}^{1} q(t) d t-\frac{2}{\lambda_{n} p} \int_{0}^{1} r(t) d t \\
& +\frac{1}{\lambda_{n}^{2} p} \int_{0}^{1} \frac{q(t)}{\lambda_{n}^{2 / p} \theta^{\prime}(t)} \frac{d}{d t}\left[S_{p}\left(\lambda_{n}^{2 / p} \theta(t)\right) S_{p}^{\prime}\left(\lambda_{n}^{2 / p} \theta(t)\right)^{p-1}\right] d t \\
& +\frac{2}{\lambda_{n} p} \int_{0}^{1} \frac{r(t)}{\lambda_{n}^{2 / p} \theta^{\prime}(t)} \frac{d}{d t}\left[S_{p}\left(\lambda_{n}^{2 / p} \theta(t)\right) S_{p}^{\prime}\left(\lambda_{n}^{2 / p} \theta(t)\right)^{p-1}\right] d t
\end{aligned}
$$

Then, using integration by parts, we have

$$
\begin{aligned}
\int_{0}^{1} \frac{q(t)}{\lambda_{n}^{2 / p} \theta^{\prime}(t)} \frac{d}{d t}\left[S_{p} S_{p}^{\prime p-1}\right] d t & =-\lambda_{n}^{-2 / p} \int_{0}^{1} G\left(\lambda_{n}^{2 / p} \theta(t)\right) \frac{d}{d t}\left(\frac{q(t)}{\theta^{\prime}(t)}\right) d t \\
& =O\left(\frac{1}{\lambda_{n}^{2 / p}}\right)
\end{aligned}
$$

where

$$
G\left(\lambda_{n}^{2 / p} \theta(x)\right)=S_{p}\left(\lambda_{n}^{2 / p} \theta(x)\right) S_{p}^{\prime}\left(\lambda_{n}^{2 / p} \theta(x)\right)^{p-1}
$$

and when $x=0,1$,

$$
G\left(\lambda_{n}^{2 / p} \theta(x)\right)=0
$$

Similarly, one can show that

$$
\int_{0}^{1} \frac{r(t)}{\lambda_{n}^{2 / p} \theta^{\prime}(t)} \frac{d}{d t}\left[S_{p} S_{p}^{\prime p-1}\right] d t=O\left(\frac{1}{\lambda_{n}^{2 / p}}\right)
$$


Inserting these values in (2.5) and after some straightforward computations, we obtain (2.4).

Theorem 2.2 For problem (1.3), (1.4), the nodal points expansion satisfies

$$
\begin{aligned}
x_{j}^{n}= & \frac{j}{n}+\frac{j}{p n^{p+1}\left(\pi_{p}\right)^{p}} \int_{0}^{x_{j}^{n}} q(t) d t+\frac{2 j}{p n \frac{2 p-2}{p}\left(\pi_{p}\right) \frac{2 p-2}{p}} \int_{0}^{x_{j}^{n}} r(t) d t \\
& +\frac{2}{\left(n \pi_{p}\right)^{\frac{p}{2}}} \int_{0}^{x_{j}^{n}} r(x) S_{p}^{p} d x+\frac{1}{\left(n \pi_{p}\right)^{p}} \int_{0}^{x_{j}^{n}} q(x) S_{p}^{p} d x+O\left(\frac{j}{n^{\frac{3 p+2}{p}}}\right) .
\end{aligned}
$$

Proof Let $\lambda=\lambda_{n}$ and integrating (2.3) from 0 to $x_{j}^{n}$, we have

$$
\frac{j \cdot \pi_{p}}{\lambda_{n}^{2 / p}}=x_{j}^{n}-\int_{0}^{x_{j}^{n}} \frac{2 r(x)}{\lambda_{n}} S_{p}^{p} d x-\int_{0}^{x_{j}^{n}} \frac{q(x)}{\lambda_{n}^{2}} S_{p}^{p} d x
$$

By using the estimates of eigenvalues as

$$
\frac{1}{\lambda_{n}^{2 / p}}=\frac{1}{n \pi_{p}}+\frac{1}{p\left(n \pi_{p}\right)^{p+1}} \int_{0}^{1} q(t) d t+\frac{2}{p\left(n \pi_{p}\right) \frac{3 p-2}{p}} \int_{0}^{1} r(t) d t+O\left(\frac{1}{n^{\frac{3 p+2}{p}}}\right)
$$

we obtain

$$
\begin{aligned}
x_{j}^{n}= & \frac{j}{n}+\frac{j}{p n^{p+1}\left(\pi_{p}\right)^{p}} \int_{0}^{x_{j}^{n}} q(t) d t+\frac{2 j}{p n^{2 p-2}} \frac{\left.2 \pi_{p}\right) \frac{2 p-2}{p}}{x_{0}^{n}} r(t) d t \\
& +\frac{2}{\left(n \pi_{p}\right)^{\frac{p}{2}}} \int_{0}^{x_{j}^{n}} r(x) S_{p}^{p} d x+\frac{1}{\left(n \pi_{p}\right)^{p}} \int_{0}^{x_{j}^{n}} q(x) S_{p}^{p} d x+O\left(\frac{j}{n^{\frac{3 p+2}{p}}}\right) .
\end{aligned}
$$

Theorem 2.3 As $n \rightarrow \infty$,

$$
l_{j}^{n}=\frac{\pi_{p}}{\lambda_{n}^{2 / p}}+\frac{2}{p \lambda_{n}} \int_{x_{j}^{n}}^{x_{j}^{n}+1} r(t) d t+\frac{1}{p \lambda_{n}^{2}} \int_{x_{j}^{n}}^{x_{j}^{n}+1} q(t) d t+O\left(\frac{1}{\lambda_{n}^{\frac{4+p}{p}}}\right) .
$$

Proof For large $n \in \mathbb{N}$, integrating (2.3) on $\left[x_{j}^{n}, x_{j+1}^{n}\right]$ and then

$$
\frac{\pi_{p}}{\lambda_{n}^{2 / p}}=l_{j}^{n}-\frac{2}{\lambda} \int_{x_{j}^{n}}^{x_{j+1}^{n}} r(t) S_{p}^{p} d t-\frac{1}{\lambda^{2}} \int_{x_{j}^{n}}^{x_{j+1}^{n}} q(t) S_{p}^{p} d t
$$

or

$$
\begin{aligned}
\frac{\pi_{p}}{\lambda_{n}^{2 / p}}= & l_{j}^{n}-\frac{2}{p \lambda_{n}} \int_{x_{j}^{n}}^{x_{j+1}^{n}} r(t) d t-\frac{1}{p \lambda_{n}^{2}} \int_{x_{j}^{n}}^{x_{j+1}^{n}} q(t) d t \\
& +\frac{2}{\lambda_{n} p} \int_{x_{j}^{n}}^{x_{j+1}^{n}} \frac{1}{\lambda_{n}^{2 / p} \theta^{\prime}(t)} \frac{d}{d t}\left[S_{p} S_{p}^{\prime p-1}\right] r(t) d t \\
& +\frac{1}{\lambda_{n}^{2} p} \int_{x_{j}^{n}}^{x_{j+1}^{n}} \frac{1}{\lambda_{n}^{2 / p} \theta^{\prime}(t)} \frac{d}{d t}\left[S_{p} S_{p}^{\prime p-1}\right] q(t) d t .
\end{aligned}
$$


By Lemma 1.1 and a similar process to that used in Theorem 2.1, we obtain that

$$
\begin{aligned}
\int_{x_{j}^{n}}^{x_{j+1}^{n}} \frac{r(t)}{\lambda_{n}^{2 / p} \theta^{\prime}(t)} \frac{d}{d t}\left[S_{p} S_{p}^{\prime p-1}\right] d t & =-\int_{j \pi_{p}}^{(j+1) \pi_{p}}\left(\frac{q(t)}{\lambda_{n}^{2 / p} \theta^{\prime}(t)}\right)^{\prime} G(\tau) \frac{d \tau}{\lambda_{n}^{2 / p} \theta^{\prime}(t)} \\
& =O\left(\frac{1}{\lambda_{n}^{4 / p}}\right)
\end{aligned}
$$

where $G(\tau)=S_{p}(\tau) S_{p}^{\prime}(\tau)^{(p-1)}$ and $\tau=\lambda_{n}^{2 / p} \theta(x)$. Similarly, one can show that

$$
\int_{x_{j}^{n}}^{x_{j+1}^{n}} \frac{q(t)}{\lambda_{n}^{2 / p} \theta^{\prime}(t)} \frac{d}{d t}\left[S_{p} S_{p}^{\prime p-1}\right] d t=O\left(\frac{1}{\lambda_{n}^{4 / p}}\right)
$$

Inserting this value in (2.7), we obtain

$$
l_{j}^{n}=\frac{\pi_{p}}{\lambda_{n}^{2 / p}}+\frac{2}{p \lambda_{n}} \int_{x_{j}^{n}}^{x_{j+1}^{n}} r(t) d t+\frac{1}{p \lambda_{n}^{2}} \int_{x_{j}^{n}}^{x_{j+1}^{n}} q(t) d t+O\left(\frac{1}{\lambda_{n}^{\frac{4+p}{p}}}\right),
$$

and by Theorem 2.1,

$$
l_{j}^{n}=\frac{1}{n}+\frac{2}{p\left(n \pi_{p}\right)^{p / 2}} \int_{x_{j}^{n}}^{x_{j+1}^{n}} r(t) d t+\frac{1}{p\left(n \pi_{p}\right)^{p}} \int_{x_{j}^{n}}^{x_{j+1}^{n}} q(t) d t+O\left(\frac{1}{n^{\frac{4+p}{p}}}\right) .
$$

\section{Reconstruction of a potential function in the differential pencil}

In this section, we give an explicit formula for a potential function. The method used in the proof of the theorem is similar to that for classical Sturm-Liouville problems $[1,8]$.

Theorem 3.1 Let $q \in L^{2}(0,1), r \in W_{2}^{1}(0,1)$ and assume $r$ that on the interval $[0,1]$ is given a priori. Then

$$
q(x)=\lim _{n \rightarrow \infty} p \lambda_{n}^{2}\left(\frac{\lambda_{n}^{2 / p} l_{j}^{n}}{\pi_{p}}-\frac{2 r(x)}{p \lambda_{n}}-1\right)
$$

for $j=j_{n}(x)=\max \left\{j: x_{j}^{n} \leq x\right\}$.

Proof Applying the mean value theorem for integrals to (2.6), with fixed $n$, there exists $z \in\left(x_{j}^{n}, x_{j+1}^{n}\right)$, we obtain

$$
l_{j}^{n}=\frac{\pi_{p}}{\lambda_{n}^{2 / p}}+\frac{2}{p \lambda_{n}} r(z) l_{j}^{n}+\frac{1}{p \lambda_{n}^{2}} q(z) l_{j}^{n}+O\left(\frac{1}{\lambda_{n}^{\frac{4+p}{p}}}\right)
$$

or

$$
q(z)=p \lambda_{n}^{2}\left(\frac{\pi_{p}}{\lambda_{n}^{2 / p} l_{j}^{n}}\right)\left(\frac{\lambda_{n}^{2 / p} l_{j}^{n}}{\pi_{p}}-\frac{2 r(z)}{p \lambda_{n}} \frac{\lambda_{n}^{2 / p} l_{j}^{n}}{\pi_{p}}-1\right)
$$

Considering (2.6), we can write that for $n \rightarrow \infty$,

$$
\frac{\lambda_{n}^{2 / p} l_{j}^{n}}{\pi_{p}}=1
$$


Then

$$
q(x)=\lim _{n \rightarrow \infty} p \lambda_{n}^{2}\left(\frac{\lambda_{n}^{2 / p} l_{j}^{n}}{\pi_{p}}-\frac{2 r}{p \lambda_{n}}-1\right) .
$$

This completes the proof.

Conclusion 3.2 In Theorem 2.1, Theorem 2.2, Theorem 2.3 and Theorem 3.1, taking $r=$ 0 , we obtain results of the Sturm-Liouville problem given in [12].

Conclusion 3.3 In Theorem 2.1, Theorem 2.2, Theorem 2.3 and Theorem 3.1, taking $p=$ 2, we obtain the results of an inverse nodal problem for differential pencil [15].

\section{Competing interests}

The author declares that they have no competing interests.

\section{Acknowledgements}

The author would like to thank the referees for valuable comments and suggestions on improving this paper.

Received: 15 October 2013 Accepted: 19 November 2013 Published: 12 Dec 2013

\section{References}

1. Law, CK, Lian, WC, Wang, WC: Inverse nodal problem and Ambarzumyan theorem for the $p$-Laplacian. Proc. R. Soc Edinb. A 139, 1261-1273 (2009)

2. Binding, P, Drábek, P: Sturm-Liouville theory for the $p$-Laplacian. Studia Sci. Math. Hung. 40, $373-396$ (2003)

3. Binding, PA, Rynne, BP: Variational and non-variational eigenvalues of the $p$-Laplacian. J. Differ. Equ. 244, 24-39 (2008)

4. Brown, BM, Eastham, MSP: Eigenvalues of the radial $p$-Laplacian with a potential on $(0,1)$. J. Comput. Appl. Math. 208, 111-119(2006)

5. Del Pino, M, Drábek, P, Manasevich, R: The Fredholm alternatives at the first eigenvalue for the one-dimensional p-Laplacian. J. Differ. Equ. 151, 386-419 (1999)

6. Drábek, P: On the generalization of the Courant nodal domain theorem. J. Differ. Equ. 181, 58-71 (2002)

7. Walter, W: Sturm-Liouville theory for the radial p-operator. Math. Z. 227, 175-185 (1998)

8. Wang, WC, Cheng, YH, Lian, WC: Inverse nodal problem for the $p$-Laplacian with eigenparameter dependent boundary conditions. Math. Comput. Model. 54, 2718-2724 (2011)

9. Hald, OL, McLaughlin, JR: Solutions of inverse nodal problems. Inverse Probl. 5, 307-347 (1989)

10. McLaughlin, JR: Inverse spectral theory using nodal points as data - a uniqueness result. J. Differ. Equ. 73, 354-362 (1988)

11. Browne, PJ, Sleeman, BD: Inverse nodal problem for Sturm-Liouville equation with eigenparameter depend boundary conditions. Inverse Probl. 12, 377-381 (1996)

12. Law, CK, Shen, CL, Yang, CF: The inverse nodal problem on the smoothness of the potential function. Inverse Probl. 15, 253-263 (1999)

13. Law, CK, Yang, CF: Reconstructing the potential function and its derivatives using nodal data. Inverse Probl. 14, 299-312 (1998); Addendum 14, 779-780 (1998)

14. Buterin, SA, Shieh, CT: Incomplete inverse spectral and nodal problems for differential pencil. Results Math. 62 , 167-179 (2012)

15. Koyunbakan, H, Yılmaz, E: Reconstruction of the potential function and its derivatives for the diffusion operator. Z. Naturforsch. A 63, 127-130 (2008)

16. Yang, XF: A solution of the inverse nodal problem. Inverse Probl. 13, 203-213 (1997)

17. Lindqvist, P: Some remarkable sine and cosine functions. Ric. Mat. XLIV(2), 269-290 (1995)

18. Koyunbakan, H: A new inverse problem for the diffusion operator. Appl. Math. Lett. 19(10), 995-999 (2006)

19. Jaulent, $M$, Jean, $C$ : The inverse s-wave scattering problem for a class of potentials depending on energy. Commun. Math. Phys. 28, 177-220 (1972)

20. Gasymov, MG, Guseinov, GS: The determination of a diffusion operator from the spectral data. Dokl. Akad. Nauk Azerb. SSR 37(2), 19-23 (1981)

21. Guseinov, GS: On spectral analysis of a quadratic pencil of Sturm-Liouville operators. Sov. Math. Dokl. 32, 859-862 (1985)

22. Hryniv, R, Pronska, N: Inverse spectral problems for energy dependent Sturm-Liouville equations. Inverse Probl. 28, 085008 (2012)

23. Nabiev, IM: The inverse quasiperiodic problem for a diffusion operator. Dokl. Math. 76, 527-529 (2007)

24. Wang, YP, Yang, CF, Huang, ZY: Half inverse problem for a quadratic pencil of Schrödinger operators. Acta Math. Sci. 31(6), 1708-1717 (2011)

25. Yang, CF: Trace formulae for the matrix Schrödinger equation with energy-dependent potential. J. Math. Anal. Appl. 393, 526-533 (2012) 
26. Koyunbakan, H: Inverse problem for quadratic pencil of Sturm-Liouville operator. J. Math. Anal. Appl. 378, 549-554 (2011)

27. Yurko, VA: Method of Spectral Mappings in the Inverse Problem Theory. Inverse and III-Posed Problems Series. VSP, Utrecht (2002)

10.1186/1687-2770-2013-272

Cite this article as: Koyunbakan: Inverse nodal problem for $p$-Laplacian energy-dependent Sturm-Liouville equation. Boundary Value Problems 2013, 2013:272

Submit your manuscript to a SpringerOpen ${ }^{\circ}$ journal and benefit from:

- Convenient online submission

- Rigorous peer review

- Immediate publication on acceptance

- Open access: articles freely available online

- High visibility within the field

- Retaining the copyright to your article

Submit your next manuscript at $>$ springeropen.com 